\title{
Pleural Effusion Due to Use of Pioglitazone: A Case Report
}

\author{
Jinhui Xue, MD, ${ }^{1}$ Wei Liu, MD, PhD, ${ }^{1}$ Fanghong Shi, MPharm, ${ }^{2}$ Jun Zheng, MD, MM, ${ }^{1}$ and Jing Ma, MD, $\mathrm{PhD}^{1}$
}

\begin{abstract}
Background: Pioglitazone is an effective treatment option in clinical practice for managing type 2 diabetes mellitus. It is challenged by a lot of adverse effects, primarily body weight gain, peripheral edema, as well as congestive heart failure.

Case Report: This is a case of a 76-year-old woman with no evidence of cardiac disease, who developed edema as well as bilateral pleural effusion after using pioglitazone. She got a weight decrease and complete resolution of the pleural effusion after stopping use of pioglitazone and rational use of diuretics.

Conclusion: Common side effects of pioglitazone have been widely reported. However, little attention was drawn on pleural effusion in diabetic patients with no pre-existing heart dysfunction. In this case, we show the use of pioglitazone results in pleural effusion, with emphasis on the clinical recognition and management.
\end{abstract}

Keywords: pioglitazone, pleural effusion, edema, thiazolidinedione, case report

\section{Introduction and Background}

$\mathrm{P}$ IOGLITAZONE, A MEMBER of thiazolidinediones, predominantly functions as an insulin sensitizer by activating nuclear peroxisome proliferator-activated receptor- $\gamma$ $\left(\right.$ PPAR- $\gamma$ ) in peripheral and hepatic tissues. ${ }^{1}$ Food and Drug Administration approved pioglitazone for the treatment of type 2 diabetes mellitus (DM) in $1999 .^{2}$ In clinical practice, the common adverse events such as fluid retention, weight gain, bone loss, and congestive heart failure have been well documented. ${ }^{3}$ However, few cases of pleural effusion have been reported in the literature in patients with normal cardiac function. ${ }^{4,5}$

\section{Case Report}

A 76-year-old woman was diagnosed with type 2 DM 20 years ago. She started injecting Novolin 30R about 5 years ago. Owing to suboptimal glycemic control, she stopped Novolin 30R and received insulin Lispro, pioglitazone (15 mg once a day) in combination with acarbose (50 mg three times a day) last year. The patient was on olmesartan to treat hypertension and aspirin to treat cerebral infarction in addition. She came to the outpatient department for progressive swelling and breathlessness 1 week ago. Pioglitazone was discontinued although she had not got other treatment for the dyspnea. She felt that breathlessness was slightly better but still suffered from edema. She had no paroxysmal nocturnal dyspnea during the course of the disease.

On arrival, her vital signs were stable with no evidence of hypoxemia. Physical examination revealed there were no extra heart sounds, jugular venous distension, hepatojugular reflux, or other classic signs of heart failure. But she displayed pitting edema of the extremities (Fig. 1A). The breath sounds were diminished in both lower lung fields and no rales were heard. Chest computed tomography (CT) showed moderate bilateral pleural effusion (Fig. 2A). Subcutaneous edema in abdominal were also found by abdominal CT scan. Her laboratory findings showed that erythrocyte sedimentation rate were elevated, but $\mathrm{C}$-reactive protein and procalcitonin were unchanged from baseline. Albumin, full blood count, serum electrolytes, liver functions, renal functions, and urine albumin creatinine ratio were in the normal range. Serum autoantibodies and antineutrophil cytoplasmic antibodies were negative. No ascites or organic abnormality was found on the abdominal CT other than gallstone. Echocardiography demonstrated normal ventricular function with an ejection fraction of $65 \%$.

\footnotetext{
${ }^{1}$ Division of Endocrinology and Metabolism, Department of Internal Medicine, Shanghai Jiao Tong University School of Medicine Affiliated Renji Hospital, Shanghai, China.

${ }^{2}$ Department of Pharmacy, Shanghai Jiao Tong University School of Medicine, Affiliated Renji Hospital, Shanghai, China.
} 

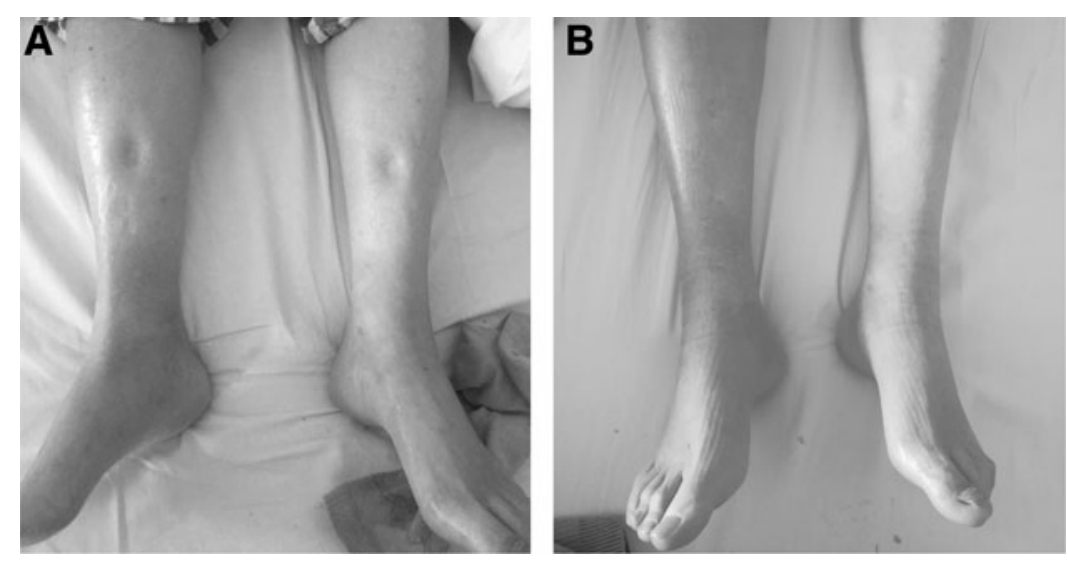

FIG. 1. (A) Pitting edema of both lower limbs at presentation, (B) improvement of edema after 7 days.

A presumptive diagnosis of pioglitazone-induced pleural effusion was made as she had no pre-existing evidence of cardiac disease or fluid overload before use of pioglitazone. Pioglitazone was discontinued and the patient was treated with furosemide $20 \mathrm{mg}$ and spirolactone $20 \mathrm{mg}$ at 12 hours intervals immediately. We also restricted her fluid and salt intake. After 7 days, She got a weight decrease from 80 to $72 \mathrm{~kg}$ (Fig. 3), and the anasarca and dyspnea began to improve (Fig. 1B). On subsequent follow-up (1 month later) in the outpatient department, she had no breathing difficulties, and her weight was dropped to $68 \mathrm{~kg}$. The chest CT showed complete resolution of the pleural effusion (Fig. 2B).

\section{Discussion}

In clinical practice, pioglitazone is the most commonly used thiazolidinedione drug that improves insulin resistance in type 2 DM by activating PPAR- $\gamma$. It can reduce the risk of stroke or myocardial infarction in patients without diabetes who had insulin resistance along with a recent history of ischemic stroke. ${ }^{6}$ Compared with glimepiride, pioglitazone results in a significantly delayed progression of coronary atherosclerosis in patients with type 2 diabetes and coronary artery disease. ${ }^{7}$ Pioglitazone also exerts favorable effects in patients with nonalcoholic steatohepatitis. ${ }^{8}$ As all the other drugs, pioglitazone has some side effects that include edema, weight gain, bone loss, congestive heart failure, and the risk of bladder cancer. ${ }^{9}$ It has been shown in a meta-analysis that pioglitazone monotherapy was significantly associated with risk of peripheral edema in type 2 DM patients. ${ }^{9}$ The incidence of edema was higher, in particular among patients who were treated with oral hypoglycemic agents or insulin at baseline. ${ }^{3}$

Renal sodium retention ${ }^{10}$ and vascular permeability ${ }^{11}$ may contribute to pioglitazone-induced edema. It has been reported edema in feet, ankles, and legs as a complication of pioglitazone in most studies, ${ }^{12}$ whereas pleural effusion is uncommon and has rarely been reported. In fact, pleural effusion, a special type of edema, is an unusual amount of fluid collected in the pleural space. In our case, we ruled out other diseases that may cause pleural effusion, such as heart disease, kidney disease, vascular disease, as well as rheumatic diseases. Insulin edema is a rare complication of insulin therapy and usually occurs in patients with type 1 DM. ${ }^{13}$ It has been mainly attributed to insulin's anabolic action, increased vascular permeability, and altered renal sodium processing. ${ }^{14}$ Insulin changes the permeability of sheep pleura and may interfere in pleural effusion formation. ${ }^{15}$ Pleural effusions have rarely been reported and were usually presented 4 weeks after use of insulin. ${ }^{13,16,17}$ In previous reports, patients were found to have pleural effusions after 5 months using pioglitazone in combination with other oral hypoglycemic agents. ${ }^{4}$ There were no reports of pleural effusion caused by the combination of insulin and pioglitazone. Our patient started insulin therapy 5 years ago, and she did not have any obvious symptoms of edema and breathing difficulties at that time. She developed symptoms of pleural effusion 1 year after the combination of pioglitazone, acarbose, and insulin. Her body weight decreased significantly before discontinuing insulin injection. Studies have shown that the edema caused by pioglitazone

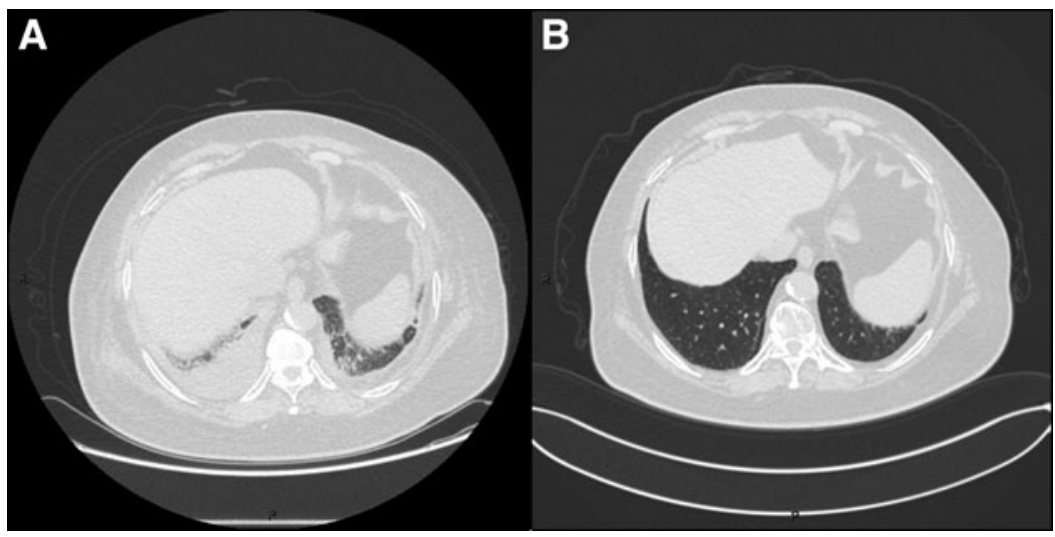

FIG. 2. Serial chest computed tomography: (A) moderate bilateral pleural effusion at presentation, (B) complete resolution of pleural effusion after 1 month. 
FIG. 3. The associated weight change during hospitalization.

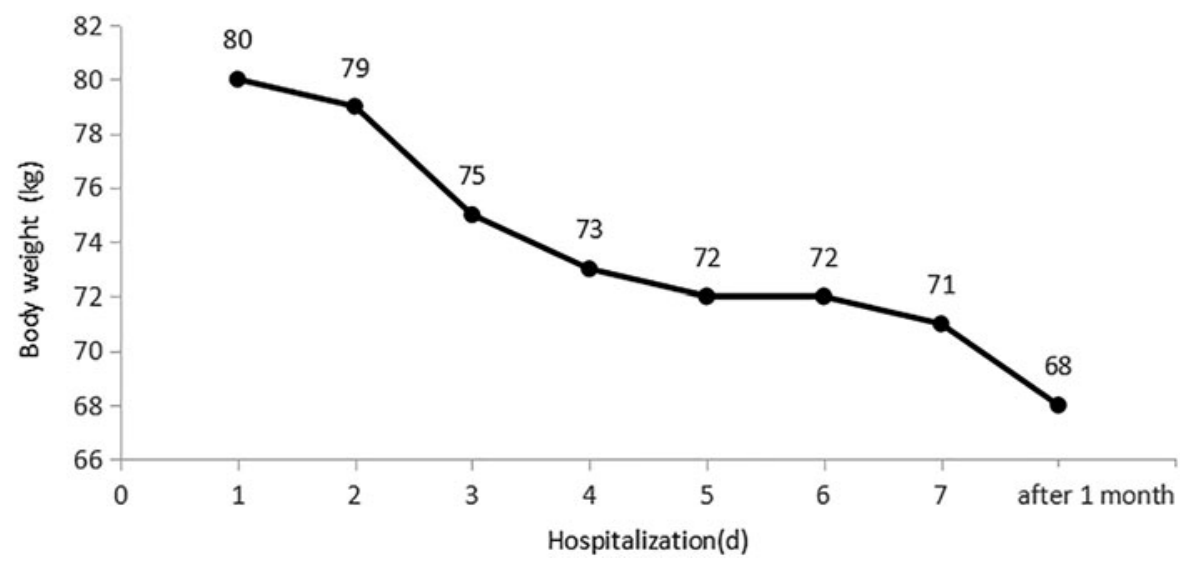

can be aggravated by combination of insulin, ${ }^{3,18}$ which may further cause pleural effusion. Although we cannot completely rule out the possibility that the interaction with insulin may contribute to the edema caused by pioglitazone and eventually lead to pleural effusion, we should think of potential possibilities of pleural effusion in patients receiving both pioglitazone and insulin.

After pioglitazone was discontinued and diuretics were used, the patient got a distinct weight decrease as well as the improvement of anasarca and dyspnea. She got a score of seven points based on the patient's Naranjo adverse drug reaction probability scale, ${ }^{19}$ which suggested that there was

Table 1. Naranjo Adverse Drug Reaction Probability Scale

Items Score

1. Are there previous conclusive reports on this reaction?

2. Did the adverse event appear after Yes (2) the suspected drug was administered?

3. Did the adverse reaction improve when the drug was discontinued, or a specific antagonist was administered?

4. Did the adverse reaction reappear when the drug was readministered?

5. Are there alternative causes (other than the drug) that could on their own have caused the reaction?

6. Did the reaction reappear when a placebo was given?

7. Was the drug detected in the blood (or other fluids) in concentrations known to be toxic?

8. Was the reaction more severe when the dose increased, or less severe when dose was decreased?

9. Did the patient have a similar reaction to the same or similar drug in any previous exposure?

10. Was the adverse event confirmed by any objective evidence?

Total score

7

Probability category: $0-3=$ doubtful; $1-4=$ possible; $5-8=$ prob able; $\geq 9=$ definite.

Don't know (0)

No (2)

Don't know (0)

Don't know (0) a probable association between the use of pioglitazone and pleural effusion (Table 1). Animal study reported the diuretic spironolactone could attenuate rosiglitazone effects on volume expansion. ${ }^{20}$ Spironolactone may be particularly effective in preventing or treating edema and pleural effusion induced by pioglitazone. Fluid and salt restriction are also necessary. ${ }^{17}$

In conclusion, for patients taking pioglitazone, especially those who use insulin at the same time, all possible side effects should be carefully monitored. When the patient has symptoms of edema and shortness of breath, clinicians should call to mind the possibility of pleural effusion.

\section{Author Disclosure Statement}

No conflicting financial interests exist.

\section{Funding Information}

This project was supported by Shanghai Municipal Education Commission-Gaofeng Clinical Medicine Grant Support (20181807), National Natural Science Foundation of China (81670728), Shanghai Pujiang Program (2019PJD027), Shanghai Renji Hospital Grant (RJKY17-01), and Shanghai Jiaotong University Grant (CHDI-2019-A-01).

\section{References}

1. Yasmin S, Jayaprakash V. Thiazolidinediones and PPAR orchestra as antidiabetic agents: From past to present. Eur J Med Chem 2017;126:879-893.

2. Miller JL. FDA approves pioglitazone for diabetes. Am J Health Syst Pharm 1999;56:1698.

3. Dormandy J, Bhattacharya M, van Troostenburg DBA. Safety and tolerability of pioglitazone in high-risk patients with type 2 diabetes: An overview of data from PROactive. Drug Saf 2009;32:187-202.

4. Chen YW, Chen YC, Wu CJ, et al. Massive bilateral pleural effusion associated with use of pioglitazone. Clin Ther 2008;30:1485-1489.

5. Munir A, Kalathil S, Nag S. Pleural effusion caused by pioglitazone: Case report. Pract Diab Int 2011;28:160.

6. Kernan WN, Viscoli CM, Furie KL, et al. Pioglitazone after ischemic stroke or transient ischemic attack. $N$ Engl J Med 2016;374:1321-1331.

7. Nissen SE, Nicholls SJ, Wolski K, et al. Comparison of pioglitazone vs glimepiride on progression of coronary atherosclerosis in patients with type 2 diabetes: The PERISCOPE randomized controlled trial. JAMA 2008;299:1561-1573. 
8. Cusi K, Orsak B, Bril F, et al. Long-term pioglitazone treatment for patients with nonalcoholic steatohepatitis and prediabetes or type 2 diabetes mellitus: A randomized trial. Ann Intern Med 2016;165:305-315.

9. Alam F, Islam MA, Mohamed M, et al. Efficacy and safety of pioglitazone monotherapy in type 2 diabetes mellitus: A systematic review and meta-analysis of randomised controlled trials. Sci Rep 2019;9:5389.

10. Horita S, Nakamura M, Satoh N, et al. Thiazolidinediones and edema: Recent advances in the pathogenesis of thiazolidinediones-induced renal sodium retention. PPAR Res 2015;2015:646423.

11. Nakamura A, Osonoi T, Terauchi Y. Relationship between urinary sodium excretion and pioglitazone-induced edema. J Diabetes Investig 2010;1:208-211.

12. Berlie HD, Kalus JS, Jaber LA. Thiazolidinediones and the risk of edema: A meta-analysis. Diabetes Res Clin Pract 2007;76:279-289.

13. Kawashima S, Kaneto H, Sakamoto K, et al. Acute progression of severe insulin edema accompanied by pericardial and pleural effusion in a patient with type 2 diabetes. Diabetes Res Clin Pract 2008;81:e18-e19.

14. Kalambokis GN, Tsatsoulis AA, Tsianos EV. The edematogenic properties of insulin. Am J Kidney Dis 2004;44: 575-590.

15. Kouritas VK, Hatzoglou C, Ioannou M, et al. Insulin alters the permeability of sheep pleura. Exp Clin Endocrinol Diabetes 2010;118:304-309.

16. Zenda T, Murase Y, Yoshida I, et al. Does the use of insulin in a patient with liver dysfunction increase water retention in the body, i.e., cause insulin oedema? Eur J Gastroenterol Hepatol 2003;15:545-549.

17. Lee $\mathrm{P}$, Kinsella J, Borkman M, et al. Bilateral pleural effusions, ascites, and facial and peripheral oedema in a 19-year-old woman 2 weeks following commencement of insulin lispro and detemir-an unusual presentation of insulin oedema. Diabet Med 2007;24:1282-1285.
18. Charbonnel B, DeFronzo R, Davidson J, et al. Pioglitazone use in combination with insulin in the prospective pioglitazone clinical trial in macrovascular events study (PROactive19). J Clin Endocrinol Metab 2010;95:21632171.

19. Naranjo CA, Busto U, Sellers EM, et al. A method for estimating the probability of adverse drug reactions. Clin Pharmacol Ther 1981;30:239-245.

20. Chang CS, Tsai PJ, Sung JM, et al. Diuretics prevent thiazolidinedione-induced cardiac hypertrophy without compromising insulin-sensitizing effects in mice. Am J Pathol 2014;184:442-453.

Address correspondence to: Jun Zheng Division of Endocrinology and Metabolism Department of Internal Medicine Shanghai Jiao Tong University School of Medicine Affiliated Renji Hospital No. 160 Pujian Road Pudong New Area Shanghai 200127

China

E-mail:13162151585@163.com

Jing $M a$

Division of Endocrinology and Metabolism Department of Internal Medicine Shanghai Jiao Tong University School of Medicine Affiliated Renji Hospital No. 160 Pujian Road Pudong New Area Shanghai 200127

China

E-mail: majing3436@163.com 